\title{
Growth Development and Maturity in Children and Adolescent: Relation to Sports and Physical Activity
}

\author{
Indranil Manna* \\ Department of Physiology, Midnapore College, Midnapore, West Bengal, India \\ *Corresponding author: indranil_manna@yahoo.com
}

Received September 01, 2014; Revised September 20, 2014; Accepted September 25, 2014

\begin{abstract}
Growth and physical maturation are dynamic processes encompassing a broad spectrum of cellular and somatic changes. The most obvious signs of physical growth are changes in overall body size. The children of the same age may differ in rate of physical growth. Ethnic variations in growth rate are also common. Thus growth norms (age-related averages for height and weight) must be applied cautiously. Physical growth, like other aspects of development, results from a complex interplay between genetic and environmental factors. Moreover, the endocrine glands also control the vast physical changes of childhood and adolescence. Although heredity remains important, environmental factors continue to affect genetic expression. Good nutrition, relative freedom from disease, and emotional well-being are essential to children's healthy development. Changes in size, proportions, and muscle strength support an explosion of new gross-motor skills. Physical activity is needed for normal growth and development, and for young people to reach their potential in muscle and bone development. Further, the psychological, social, and physical development process project powerful influences on sport participation. Sports scientists, physicians and physical educators must be familiar with the normal patterns of growth and development of the child and adolescent. This allow finding out deviations during the pre-participation examination, guiding children into appropriate activities, aiding them in setting realistic goals concerning sports participation. It also provides guidance to the community and coaches in the design of safe and effective training programme.
\end{abstract}

Cite This Article: Indranil Manna, "Growth Development and Maturity in Children and Adolescent: Relation to Sports and Physical Activity.” American Journal of Sports Science and Medicine, vol. 2, no. 5A (2014): 48-50. doi: 10.12691/ajssm-2-5A.

\section{Introduction}

Growth refers to measurable changes in size, physique and body composition, and various systems of the body, whereas maturation refers to progress toward the mature state. The processes of growth and maturation are related, and both influence physical performance. There are three broad stages of development: early childhood, middle childhood, and adolescence. The definitions of these stages are organized around the primary tasks of development in each stage, though the boundaries of these stages are malleable [1]. Growth in stature is rapid in infancy and early childhood, rather steady during middle childhood, rapid during the adolescent spunt, and then slow as adult stature is attained [1,2]. This pattern of growth is generally similar for body weight and other dimensions with the exception of subcutaneous fat and fat distribution [1,2]. The growth rate of stature is highest during the first year of life then gradually declines until the onset of the adolescent growth spurt (about 10 years in girls and 12 years in boys). With the spurt, growth rate increases, reaching a peak at about 12 years in girls and 14 years in boys, and then gradually declines and eventually ceases with the attainment of adult stature $[2,3]$. In adolescents the skeleton first grows in size and length, after which it gains in density and strength $[2,3]$.
The principal sites of growth before the start of rapid adolescent growth are in the legs and arms [2,3]. During the adolescent growth spurt, the trunk grows most rapidly $[2,3]$. The long bones of the arms and legs increase their length by the activity of specialized cells located in a socalled growth plate at either end of the shaft of the long bones. As growth nears completion in later adolescence, the growth plate ceases its function, fuses firmly with the shaft of the long bone [2,3].

\section{Changes in Physical Abilities during Childhood and Adolescence}

At the time of childhood, as boys and girls growresulting in longer levers and increased muscle tissue-both have the potential to increase their strength. Boys and girls show similar ability to perform motor skills prior to puberty $[4,5]$. In general, boys develop greater strength and thus surpass girls in the performance of most sportrelated skills. During adolescence, males show a steady increase in performance and endurance that extends into early adulthood. There are dissimilarities among the girls. There has been a tendency for girls' performance to reach a plateau around the time of puberty (approximately 13 years of age) and decline thereafter [4,5]. Because of physical changes that accompany adolescence, such as 
increases in fat, girls have a disadvantage for motor performance [4,5]. Like other aspects of motor skill, strength shows a steady increase during childhood, with boys being slightly stronger than girls. Boys continue to improve during adolescence, whereas girls' strength level off and then tend to decrease [4,5]. In boys there is a delay, on the average, of at least fourteen months between the period of the most rapid gain in height and the most rapid gain in muscle weight. The adolescent male who is nearing the completion of his rapid gain in height will have little muscle tissue and strength potential for the next year or two $[4,5]$. Thus, the adolescent male is not as strong as his stature might suggest. The best information available indicates that prior to the age of 14 years (and the production of the male sex hormone testosterone), weight training cannot be expected to result in any worthwhile gains in either muscle development or strength $[4,5]$. In addition, weight training for preadolescent boys is an activity with high injury risk if not properly supervised.

\section{Sport Participation and Physical Maturity}

The body structure and a variety of basic functions that relate to athletic performance undergo striking change during the early years of adolescence. The age at which children are physically ready for many types of sports also vary greatly. It is important to identify early-maturing and late-maturing individuals if they are to be directed into appropriate sport experiences [5,6]. Early maturation in boys is an advantage in some sports, but the opposite applies in girls. There is an apparent delay in maturity in sports where females who maintain preadolescent physique seem to have an advantage [5,6]. Successful female athletes display physical characteristics that favor good performances successful young female athletes have similar somatotypes to older successful athletes [5,6]. There is a trend towards increase linearity in these athletes and this linear physique characterizes the physical attributes of late maturing girls [5,6]. Early maturing girls undergo a socialization process which does not motivate them any more to excel in physical exercise. On the other hand, late-maturing girls tend to be socialized into sports participation. Late-maturing girls are older chronologically when they attain menarche and have not yet experienced the social pressures regarding competitive athletics for girls and/or are more able to cope with the social pressures $[5,6]$. The late mature athletes have less strength, endurance, and skeletal maturity and lower motor skills than their average peers $[5,6]$. The late mature athletes have increased risk of injury, with his/her undeveloped muscles and immature skeleton [5,6]. More importantly, playing with and competing against larger, stronger, and more mature athletes, the late maturer have been a less skilled athlete, and is a prime candidate to drop out at the earliest opportunity. Parents and coaches should know the implications of delayed adolescent development, and they should develop their expectations accordingly $[5,6]$.

\section{Motor Skill Development}

Motor skills develop in the first eighteen years of life, although in girls their development tends to stabilize around puberty [6,7]. Strength and power rapidity increase in proportion to muscle mass under the influence of hormonal activity. The daily use of motor activities, games and physical education must allow children to acquire a set of motor skills i.e., gross motor skill development $[6,7,8]$. During middle childhood, children continue to build on and improve gross motor skills; the large-scale body movement skills such as walking and running that they first learned during earlier developmental stages. In general, boys develop these skills slightly faster than do girls, except for skills involving balance and precise movements such as skipping, jumping and hopping. At this age, children run faster and jump higher than previously possible. These figures are average for children of this age range and will not apply to individual children. Middle-childhood-aged children also refine their control over gross motor skills, learning to master where they hop, skip, throw, and jump. They are able to gain this improved control and coordination due to increases in their flexibility, balance, and agility $[6,7,8]$. Children at this age also learn how to synchronize the movement of their body's various parts, allowing for the development of smoother, more coordinated whole-body movement routines such as are needed for participating in organized sports. Due to their progress with regard to the growth and maturity of motor, cognitive, and social skills, many children will become capable and competitive participants on sports teams.

\subsection{Development of Strength}

Motor power rises gradually over the course of the growth process depending on the increase in body mass. Before puberty, maximum strength in boys and girls remains relatively similar. Improved nerve activation and increased muscle mass (hypertrophy) are the main explanation for the increase in strength $[6,7,8]$. Before puberty, improvement concerns mainly nerve activation. Other mechanisms, including improved elastic energy release, intensified excitation-contraction coupling, and improvement in strength transmission to different bone levers, are also involved $[6,7,8]$. This rise in strength has an impact on the capacity for motor skill performance in fitness activities and in the prevention of injuries during such activities. Since the increase in testosterone production in adolescent children is markedly higher in boys than girls, boys become stronger faster and to a higher degree $[6,7,8]$. Whole-body activities are more important and beneficial than the same exercises used for post-pubescent athletes. The development of these qualities quickens during the post-puberty period $[6,7,8]$.

\subsection{Development of Aerobic Power}

Aerobic power increases with age during childhood in both sexes and is quite similar. Girls hardly differ from boys in the prepubertal period but, from the age of 14 years on their aerobic power is significantly lower by about $15 \%[9,10]$. The maximal aerobic performance capacity in girls reaches a plateau from 14 years onwards while in boys it increases up to the age of 18 years $[9,10]$. Thus, even though the aerobic capacity is fully developed aerobic performance continues to improve. That is because other growth factors, such as larger levers, greater musculature, etc. are still developing and govern the 
effectiveness and mechanical efficiency of aerobic activities $[9,10]$. Endurance training has been shown not to effect aerobic capacity before 11 years. After the age of 12 years, an improvement in $\mathrm{VO}_{2 \max }$ has been shown in males $[9,10]$. This suggests that there is an increased trainability of the heart and circulatory system around puberty in males $[9,10]$. It takes a lot of intense aerobic training to produce shifts in aerobic factors in children. $\mathrm{VO}_{2 \max }$ improvements are similar to those reported for adults when the training volumes and intensities are very high. Short-term training programs have no significant effect on improving $\mathrm{VO}_{2 \max }$ in pre-pubertal children [9,10]. The improvements are probably due to motor coordination and running technique $[9,10]$. In pre-pubertal children, the gains from endurance training largely result from improvements in mechanical efficiency not a large change in physical aerobic power. Thus, for endurance improvements, an emphasis on the techniques of performance is more beneficial than the programming of assumed physiological stimulations of training.

\subsection{Development of Anaerobic Capacity}

Unlike aerobic capacity, the anaerobic capacity of children expressed per $\mathrm{Kg}$ of body weight is much smaller than adults. It is lowest in children and increases progressively with age in both boys and girls $[6,7,8]$. The ratio of aerobic: anaerobic metabolism contribution to exercise differs between children and adults. Children are best suited to adapt to aerobic exercises. Frequent and stressful stimulation of anaerobic metabolism will be particularly fatiguing and if overdone, could be harmful $[6,7,8]$. Children may fatigue rapidly in anaerobic work when compared to their response to endurance work.

\section{Conclusion}

Physical activity, whether through informal or organized sports, is important for optimal health, growth and development of children. Physical and physiological process influences on sports participation and performance in sports. The aspects of development influencing sport participation among children and adolescents. Understanding athlete development and outcome of sport participation is a key to effective coaching and teaching of young athletes, and helps coaches work more effectively with the young athletes. The maturation and environmental factors influence the progressive development of children and adolescents. Keeping these principles in mind, the sport talent of young athletes can be developed. Therefore, these factors need to accommodate as part of the effective sports skill instruction. These developmental issues help coaches to use their knowledge for development of effective training programme for children and adolescents. This special issue of the American Journal of Sports Science and Medicine focused on the current understanding of Growth and Development in Children and Adolescent in Relation to Sports and Physical Activity.

\section{References}

[1] Neoklis A, Georgopoulos, Kostas B. et al. Growth, pubertal development, skeletal maturation and bone mass acquisition in athletes. Hormones. 2004; 3(4): 233-243.

[2] Tanner JM. Growth at adolescence, 2nd Ed. Oxford: Blackwell. 1962.

[3] Malina RM, Bouchard C, and Bar-Or O. Growth, Maturation and Physical Activity, 2nd Ed. Human Kinetics, Champaign, IL. 2004.

[4] Rogol AD, Clark PA, Roemmich JN. Growth and pubertal development in children and adolescents: effects of diet and physical activity. Am J Clin Nutr. 2000; 72(2): 521S-528S.

[5] Byrne NM, and Hills AP. The importance of physical activity in the growth and development of children. In Hills AP, King NA, and Byrne NM (Ed.) Children, obesity and exercise: prevention, treatment and management of childhood and adolescent obesity. Routledge, London, 2007; 50-60.

[6] DErcole AA, D Ercole C, Gobbi M, Gobbi F. Technical, perceptual and motor skills in novice-expert water polo players: an individual discriminant analysis for talent development. J Strength Cond Res. 2013; 27(12):3436-3444.

[7] Roemmich JN, and Sinning WE. Weight loss and wrestling training: Effects on nutrition, growth, maturation, body composition, and strength. J Appl Physiol. 1997; 82, 1751-1759.

[8] Pahkala K, Hernelahti M, Heinonen OJ et al. Body mass index, fitness and physical activity from childhood through adolescence. Br J Sports Med. 2013; 47(2): 71-77.

[9] Hayes HM, Eisenmann JC, Pfeiffer K, Carlson JJ. Weight status, physical activity, and vascular health in 9- to 12-year-old children. J Phys Act Health. 2013; 10(2): 205-210.

[10] Rowland TW. Effect of prolonged inactivity on aerobic fitness of children. J Sports Med Phys Fit. 1994; 34, 147-155. 